\title{
A FORWARD DETECTOR FOR THE DO AREA AT FERMILAB
}

\author{
Michael J. Longo \\ University of Michigan, Ann Arbor, MI 48109
}

\section{ABSTRACT}

About $90 \%$ of the energy from a $\sqrt{s}=2000 \mathrm{GeV} \overrightarrow{\mathrm{pp}}$ collision goes out at angles $<2^{\circ}$ in the laboratory. We propose a detector for the DO a rea which emphasizes tracking and calorimetry down to the smallest practical angles. A detector of this type is essential for studying the general features of collisions at Collider energies, particularly the energy flow, multiplicity and inelastic cross section. It will also play a very important role in selecting hard collisions which will reduce the background for new physics in a central detector.

Let me first try to educate you as to where the "action" is at the Fermilab collider. If we identify "action" with energy, it is clear that almost all the action is very forward - at angles not covered by the presently contemplated detectors at Fermilab.

Everything I have to say is, of course, based on a model of what will happen at collider energies. (I'm indebted to Tom Gaisser for a program to simulate high-energy collisions. To a large extent I'm just reemphasizing points he has already made about energy flow. ${ }^{1}$ ) Let me, therefore, say a little about models. The most naive model to extrapolate from ISR to collider energies is Feynman scaling

$$
E \frac{d^{2} \sigma}{d p_{L}} d p_{T}=f\left(x, p_{T}\right)
$$

where $p_{L}$ is the longitudinal and $p_{T}$ the transverse momentum and $x \cong p L /(\sqrt{s} / 2)$. Feynman scaling implies that we can take an event at the ISR energy $\sqrt{s_{0}}$ and scale it to a higher energy $\sqrt{s}$ by simply scaling the longitudinal momenta of the particles by $\sqrt{\mathrm{s} / \mathrm{s}_{0}}$, or

$$
\frac{p_{L}(s)}{p_{L}\left(s_{0}\right)}=\frac{\sqrt{s}}{\sqrt{s_{0}}}
$$

Feynman scaling is known not to work very well at very high energies 2,3 .

Another type of scaling is statistical scaling which says that each secondary, on the average, carries off $1 / n_{S}$ of the total energy where $n_{S}$ is the mean multiplicity. This leads to a scaling 0094-243X/82/850318-07\$3.00 Copyright 1982 American Institute of Physics 
of the type

$$
\frac{p_{L}(s)}{p_{L}\left(s_{0}\right)}=\frac{\sqrt{s} / n_{s}(s)}{\sqrt{s_{0}} / n_{s}\left(s_{0}\right)}
$$

If the multiplicity follows a power law in $\sqrt{s}, n_{S}=A(\sqrt{s})^{\alpha}$, statistical scaling implies

$$
\frac{p_{L}(s)}{p_{L}\left(s_{0}\right)}=\left(\frac{\sqrt{s}}{\sqrt{s}}\right)^{1-\alpha_{n}}
$$

where $\alpha_{n} \approx 0.43$ (Ref. 2). Feynman scaling is clearly a special case of this with $\alpha_{n} \equiv 0$.

The correct scaling law is thought to lie somewhere between the Feynman and statistical extremes. A somewhat more general kind of scaling law than Eq. (1) has therefore been discussed by Wdowczyk and Wolfendale ${ }^{2}$, Gaisser ${ }^{1}$, and others. This has the form

$$
E \frac{d^{2} \sigma}{d p_{L}} \frac{\sigma}{d p_{T}}=\left(\frac{s}{s_{0}}\right)^{\alpha} f\left[\left(\frac{s}{s_{0}}\right)^{\alpha} x, p_{T}\right]
$$

where $\alpha$ now is a parameter to be determined from data. Presumably,

$$
\underset{\begin{array}{c}
\alpha_{n} \\
\text { (statistical } \\
\text { scaling) }
\end{array}}{ }>\alpha>\underset{\begin{array}{c}
\text { (Feynman } \\
\text { scaling) }
\end{array}}{0}
$$

I now return to my original question: Where is all the "action" in $\sqrt{\mathrm{s}}=2000 \mathrm{GeV}$ p collisions? Given a Monte Carlo program which incorporates the scaling 1 aw (5) and a reasonable value for $\alpha$, we can generate $\sqrt{\mathrm{s}}=2000 \mathrm{GeV}$ collisions starting with ISR data.

From an experimentalist's point of view the results are nicely summarized in Fig. 1. This shows for three values of $\alpha$ the fraction of the energy which goes outside an angle $\theta_{\text {min }}$. For the $\alpha=0.19$ curve only I show what happens if you include the leading nucleons. In this model they are given on the average half the energy; they are assumed to obey Feynman scaling so their contribution does not depend on $\alpha$.

The meaning of these curves can be illustrated by an example. It is hoped that CDF will have a forward detector which goes down to $2^{\circ}$ or so in either hemisphere. If Feynman scaling is correct $(\alpha=0)$, the entire CDF detector on the average would only see about $2 \%$ of the total energy from a collision! If $\alpha=0.19$, a reasonable 


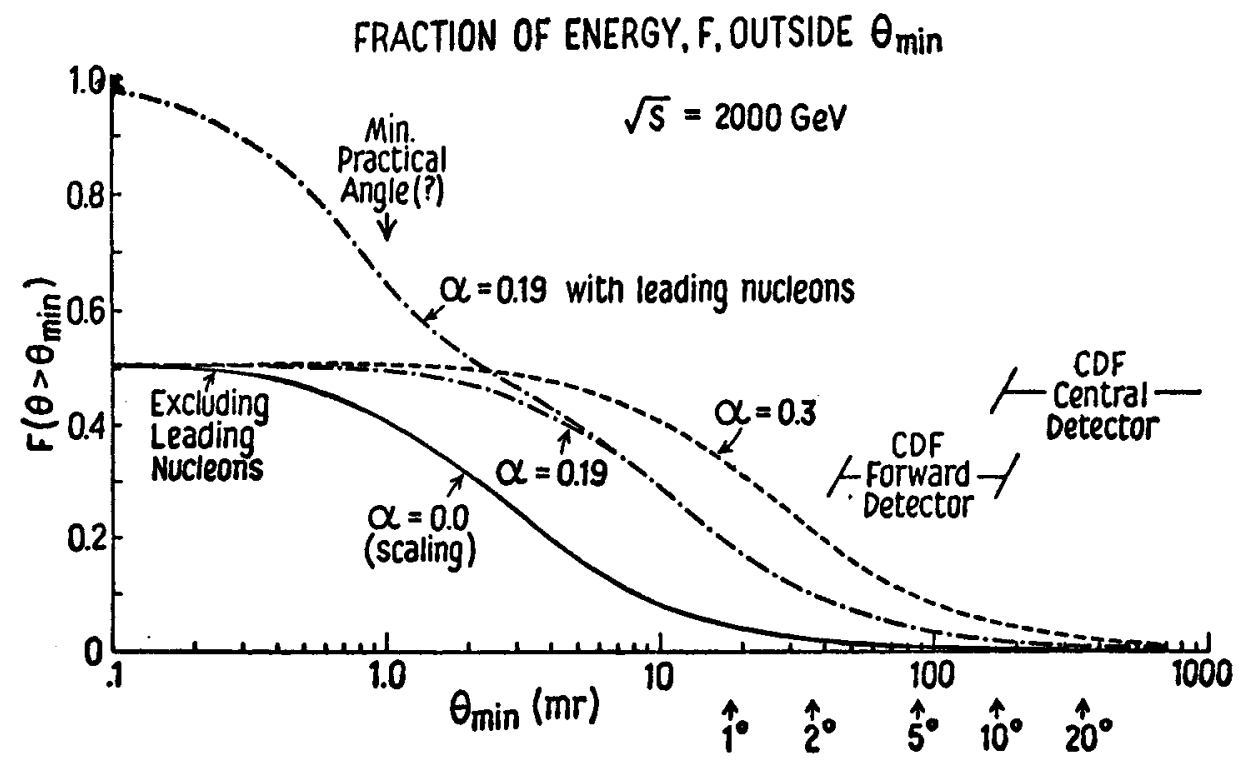

Figure 1 - Fraction of energy collected in a detector which covers $\theta>\theta_{\min }$ vs. $\theta_{\min }$.

value based on cosmic ray data ${ }^{2}$, it would see about $10 \%$ of the energy.

This strikes some people as a serious shortcoming of the CDF detector and raises the question: Can one do better? The straight sections at the Fermilab collider are about $50 \mathrm{~m}$. long. At the ISR, detectors can be placed as close as $0.7 \mathrm{~cm}$ from the circulating beam. This combination would give $\theta_{\min } \sim 0.7 \mathrm{~cm} / 25 \mathrm{~m} \cong$ $0.3 \mathrm{mr}$. A more conservative design might be to take $\theta_{\min }=2$ $\mathrm{cm} / 20 \mathrm{~m}=1 \mathrm{mr}$. This is shown in Figure 1 as the "Min. Practical Angle". If $\alpha$ isn't too close to 0 , a calorimeter that could see down to $1 \mathrm{mr}$ would collect almost all the energy except that carried by the leading nucleons.

This gives a good idea what we need to do and suggests a detector like that shown in Fig. 2. It is almost the logical complement of CDF. The detectors are stretched out along the beam line over the entire length of the straight section. The emphas is is on multiplicity counting and calorimetry. The interaction region would be surrounded by wire chambers and counters. Each of the six calorimeters would be preceeded by wire chambers to track particles and measure multiplicity. The detector is modest in size. Typical dimensions of the calorimeter are $\sim 1 \mathrm{~m}$. (Note that transverse dimensions are exaggerated tenfold in the figure.) 


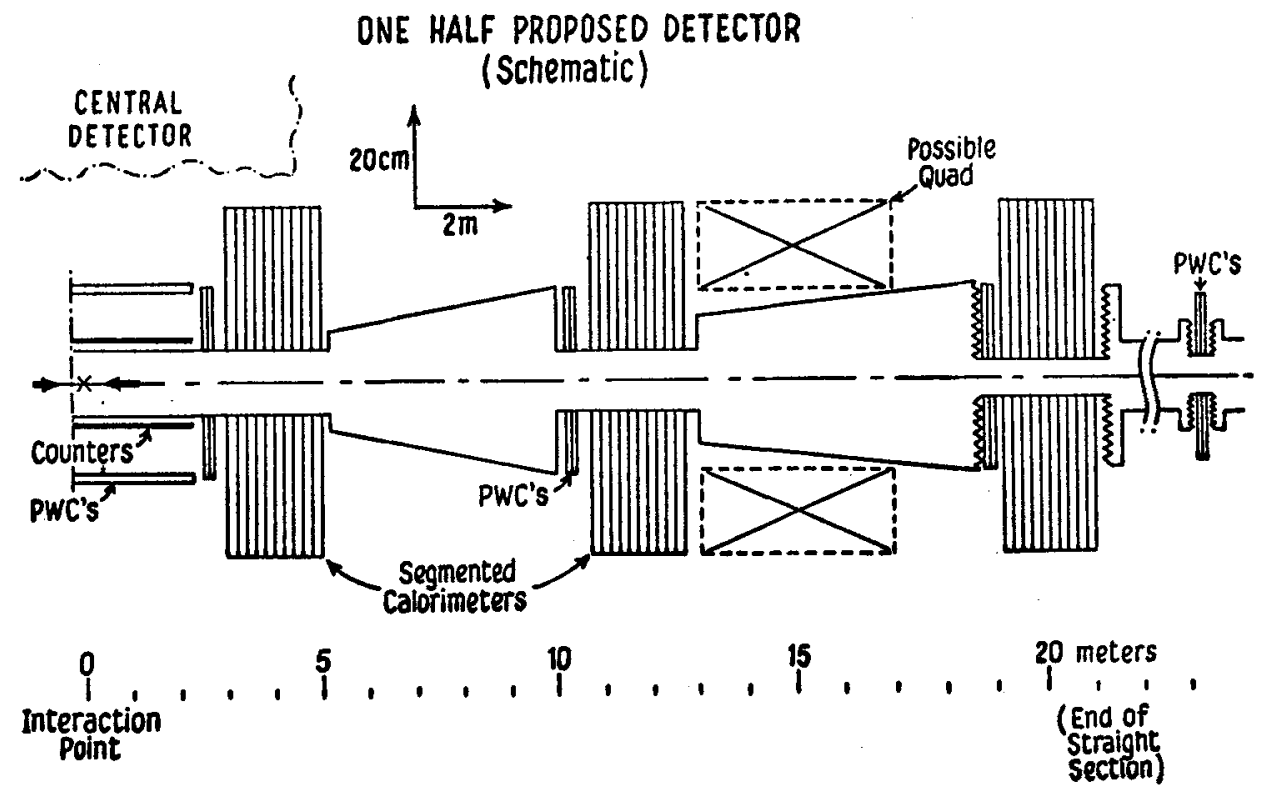

Figure 2 - Schematic of possible experimental arrangement.

The farthest calorimeters have to be mounted in such a way that they can be moved in to $<2 \mathrm{~cm}$ from the circulating beam once the beams are stable.

As far as physics this detector could do on its own, some of the more obvious things are the following:

(1) Multiplicity vs s - This is a basic measurement. Cosmic ray data from the Japan-Brazil group suggest a new threshold near $\sqrt{\mathrm{s}} \cong$ $500 \mathrm{GeV}$. (See inset to Fig. 3 which is taken from G. Goggi, CERN-EP/81-08.)

(2) ojnel vs s - Again cosmic ray data suggest a sudden increase in absorption length above $\sqrt{\mathrm{s}} \cong 500 \mathrm{GeV}$ as shown in Fig. 4 .

(3) Centauro events - These have been discussed at length by many people. These also seem to be restricted to $\sqrt{\mathrm{s}}>600 \mathrm{GeV}$, perhaps just out of reach of the CERN collider. (See Fig. 3).

(4) Energy flow measurements - As discussed earlier in the introduction, these are essential to understanding the general features of interactions at very high energies. As Gaisser has emphasized ${ }^{1}$, these data are important in our attempts to model cosmic ray interactions at extremely high energies which is necessary in answering very basic questions like the composition of the high energy component. 


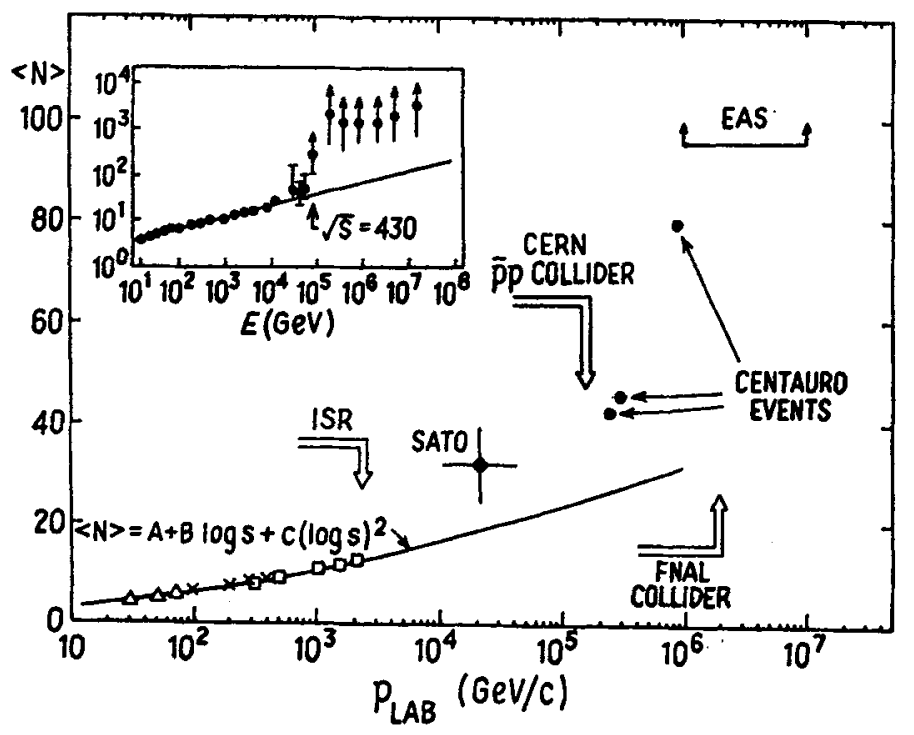

Fig. 3. Charged multiplicities from accelerators, ISR, and cosmicray experiments (from G. Goggi, CERN-EP/81-08).

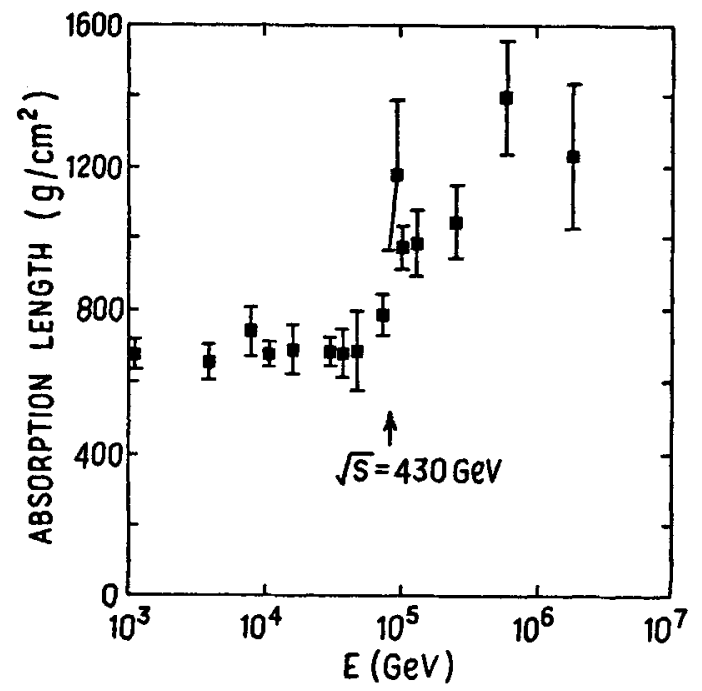

Fig. 4. The Tien-Shan anomaly in the absorption length for cosmic ray showers in a calorimeter. (From G. Goggi, CERN-EP/81-08). 
(5) S. Brodsky at this conference pointed out that calorimetry down to small angles will allow the identification of an interesting class of events in which all the valence quarks in the $P$ annihilate with the antiquarks in the $\bar{P}$ leave no leading particles.

Obviously much of the above will be well studied at CERN long before the Fermilab collider is operational. However, it will be important to extend the measurements from $\sqrt{\mathrm{s}}=540$ to $\sqrt{\mathrm{s}}=2000 \mathrm{GeV}$, and the CDF is not well suited for doing most of the above physics.

In addition to the above, there are some less obvious, but perhaps more important, uses of such a detector. I assume we' $d$ be in DO along with some central detector, presumably built by another group. Our forward detector would provide a significant tool to enhance new physics signals in the central detector. This might prove crucial in separating objects like the $W$ from the dominant background.

Broadly speaking, to produce these massive states requires a hard collision between $a \mathrm{q}$ and $\bar{q}$ with the maximum possible $s$, the center-of-mass energy squared of the $q \bar{q}$. Events of this type are characterized by:

(1) high multiplicity

(2) little energy going down beam pipes.

Our detector would be uniquely capable of answering these questions on an event-by-event basis. Selecting events which satisfy these criteria should significantly reduce the background in searches for the $w, t, \ldots$

Carrying this line of reasoning somewhat farther, we may be able to make studies of hadron-hadron interactions a lot more like $\mathrm{e}^{t} \mathrm{e}^{-}$. Referring to Fig. 1, if calorimeters cover all angles down to about $1 \mathrm{mr}$, all the energy except that carried by the leading particles is contained. To the extent that we can identify the leading particles with the fragments of the "wounded" nucleons from a hard $q q$ collision, the total energy seen in the calorimeters gives $\sqrt{s}$, the c.m. energy of the qq which make the hard collision. If you now plot your favorite indicator of new physics, such as the rate

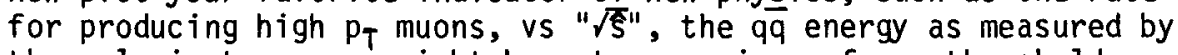
the calorimeters, you might hope to see signs of new thresholds such as in Fig. 5 . 


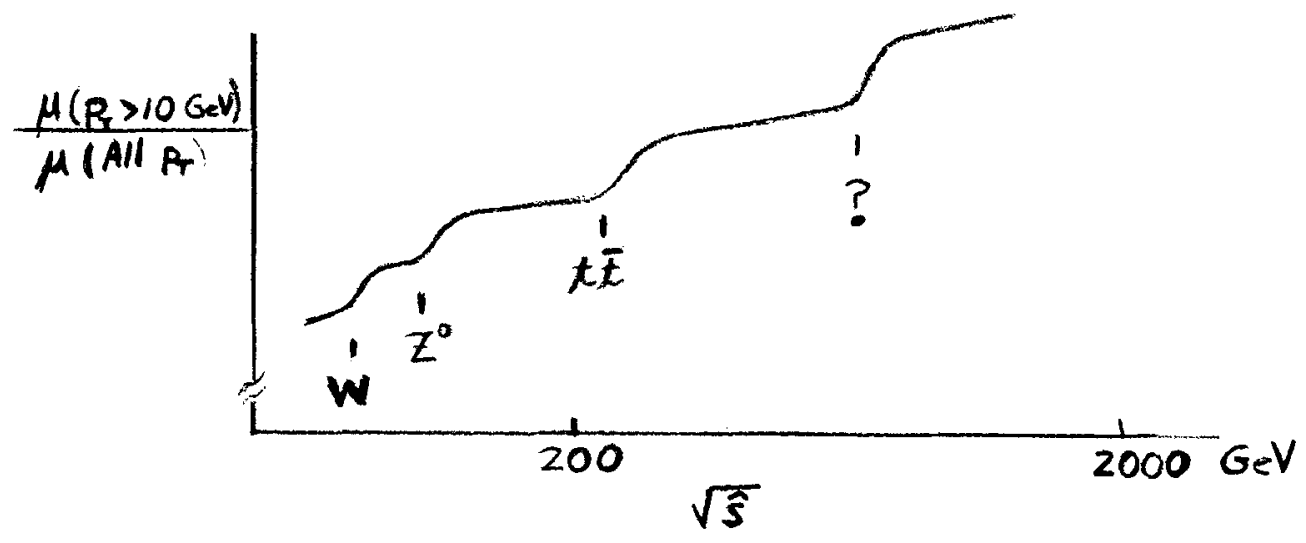

Figure 5 - Idealized variation of large $\mathrm{P}_{T}$ muon yield with $\sqrt{\mathrm{S}}$.

Figure 5 certainly is overly optimistic, but even having a rough measure of $\hat{s}$ for each interaction could be an important new technique in collider physics.

\section{REFERENCES}

1. T.K. Gaisser, Phys. Lett I00B, 425 (1981).

2. J. Wdowczyk and A.W. Wolfendale, Nuovo Cim. 54A, 433 (1979).

3. K. Guettler et al., Phys. Lett. B64, 111 (1976).

R. Schindler et al., Phys. Rev. Lett. 33,862 (1974). 